\title{
Scandium: a key element for understanding Am stars
}

\author{
F. LeBlanc ${ }^{1}$ and G. Alecian ${ }^{2}$ \\ 1 Département de Physique et d'Astronomie, Université de Moncton, Moncton, NB, E1A 3E9, Canada \\ e-mail: francis.leblanc@umoncton.ca \\ 2 LUTH (Observatoire de Paris - CNRS), Observatoire de Meudon, 92195 Meudon Cedex, France
}

Received 10 April 2007 / Accepted 19 October 2007

\begin{abstract}
Context. Atomic diffusion is believed to cause the abundance anomalies observed in AmFm stars. However, the detailed process has still not been well-established. For instance, two possible scenarios for the diffusion theory are presently envisaged. They differ mainly by the depth from which the abundance anomalies emanate. The first scenario predicts that the abundances are modified in the superficial regions of the star, just below the hydrogen convection zone. The second scenario predicts that a much deeper extension of the mixing zone exists due to the convection caused by Fe accumulation in regions below the hydrogen convection zone.

Aims. We calculate much more accurate radiative accelerations of Sc than previously, to better understand the observed abundance anomalies of this element. We believe that it is a key element to use as a diagnostic tool for understanding AmFm stars.

Methods. The method employed to obtain these radiative accelerations is based on an interpolation from the parameters of the socalled SVP parametric method.

Results. The radiative accelerations, shown here in a typical Am stellar model, are discussed in light of the observed anomalies of Ca and Sc. Our results suggest that the deeper mixing scenario is not entirely satisfactory: the mixing zone should be deeper than what is predicted by recent models to account for observed Sc underabundances. Our results seem more compatible with the scenario where the abundances anomalies are created in the superficial regions. However, only detailed evolutionary modelling with mass loss and diffusion of all important species, including $\mathrm{Ca}$ and $\mathrm{Sc}$, with accurate radiative accelerations, will be able to give more insight into where the source of these anomalies occur in AmFm stars.
\end{abstract}

Key words. diffusion - stars: abundances - stars: chemically peculiar

\section{Introduction}

Among the main observational peculiarities of Am stars are the superficial underabundances of $\mathrm{Ca}$ and $\mathrm{Sc}$, as well as an overabundance of iron-peak elements (e.g. Preston 1974). The classical explanation of the AmFm star phenomenon is that these stars, which are slow rotators, are supposed to have smaller large-scale (mixing) motions in their envelope than normal main sequence stars. Since these stars are almost always in binary systems, their slow rotation is believed to be due to tidal forces (Abt 1961). The relative stability of their envelope allows for a large proportion of He atoms to sink towards the deeper regions due to gravitational settling, leading to the disappearance of the He convection zone. The abundance anomalies were then thought to be produced by the diffusion process (Michaud 1970) at the bottom of the hydrogen convection zone (Watson 1971; Smith 1971; Michaud et al. 1983; Alecian 1996). At that depth, Ca is mostly in its Ar-like configuration so its radiative acceleration is weak because of its noble gas configuration, thus leading to its underabundance in the superficial hydrogen convection zone. The radiative acceleration $\left(g_{\mathrm{rad}}\right)$ is due to the momentum transfer from photons to atoms during photoabsorption. The value of $g_{\mathrm{rad}}$ of a given element depends on its absorption cross-section, on its abundance and on the local physical conditions of the plasma (e.g. Alecian \& LeBlanc 2000). The diffusion process that can create over or underabundances at various depths is a non linear, timedependent process that can affect the structure and the evolution of stars.
Numerical modelling of $\mathrm{Ca}$ and Sc time-dependent diffusion in AmFm stars was carried out by Alecian (1996). This modelling corresponds to the scenario first proposed by Watson (1971), but including a bulk velocity due to mass loss (as considered for $\lambda$ Bootis stars by Michaud \& Charland 1986). Superficial abundances of these elements appear to depend closely on the age of the star, on the mass loss rate, and they are very sensitive to the position of the bottom of the superficial mixing zone (hydrogen convection zone plus overshooting). In these computations, the stellar model was static and no structure evolution was considered (the star was kept at the same position on the main sequence), only diffusion was time-dependent.

Recent evolutionary models including the effect of the diffusion of the elements show that a Fe convection zone can occur due to its accumulation at certain depths (Richard et al. 2001; Richer et al. 2000), and suggest that the abundance anomalies emanate from deeper layers (at temperatures of approximately $200000 \mathrm{~K}$ ). At that depth, a large proportion of $\mathrm{Ca}$ is also in a noble gas configuration (Ne-like) which explains its observed underabundance. However, these computions are done without mass loss. Mass loss has two effects: it smooths the abundance inhomogenietes, and it shifts the abundance stratifications produced by diffusion towards shallower layers in the star (Michaud \& Charland 1986; Alecian 1996). Therefore, mass loss may affect iron accumulation and thus the Fe convection zone predicted by these evolutionary models.

The study of $\mathrm{Ca}$ diffusion is relatively easy because its atomic data are rather well known. Indeed, a good knowledge 
of the atomic data is essential since radiative accelerations are, with gravity, among the main ingredients in the diffusion equation. The situation for $\mathrm{Sc}$ is quite different. For instance, both the OPAL opacities (e.g. Rogers \& Iglesias 1992) and the Opacity Project (hereafter OP, Seaton et al. 1992) do not include scandium in their databases. The Kurucz (1990) data include only the first nine Sc ions. The detailed study of Sc diffusion has never been done. Alecian (1996) calculated the $g_{\mathrm{rad}}$ of Sc based on the parametric method of Alecian \& Artru (1990a,b). But, as mentioned in his study, the calculated $g_{\text {rad }}$ are not accurate enough since extrapolations had to be done to complete the data for highly ionized Sc ions. In this paper, we aim to calculate the $g_{\mathrm{rad}}$ of Sc by an interpolation method based on the parametric form of the $g_{\text {rad }}$ formulae of Alecian \& LeBlanc (2002) and LeBlanc $\&$ Alecian (2004) (hereafter respectively Papers I and II).

Because scandium underabundance, along with the one of calcium, characterizes AmFm stars, it is a key element to better understand the AmFm phenomenon. Scandium is in an ionisation state with noble gas configuration in layers close to the bottom of the $\mathrm{H}$ convection zone, and close to the bottom of the Fe convection zone predicted by Richer et al. (2000), but not at the same positions as for the corresponding calcium configurations. Any model of AmFm stars should be able to explain underabundances for both elements. Therefore, the study of Sc diffusion could possibly shed light on which one of the two diffusion scenarios, diffusion below the $\mathrm{H}$ convection zone while including mass loss (Alecian 1996; hereafter scenario A), and diffusion below the Fe convection zone (Richer et al. 2000; hereafter scenario $B$ ), is more likely to explain the abundance anomalies of these stars.

In Sect. 2, we present $g_{\text {rad }}$ calculations for Sc while using an interpolation method related to the new parametric formulae described in Papers I and II. In Sect. 3, we discuss our $g_{\text {rad }}$ with respect to the observed abundance anomalies of $\mathrm{Ca}$ and $\mathrm{Sc}$ in AmFm stars. A short conclusion follows.

\section{Radiative acceleration calculations}

\subsection{Method used for $g_{\text {rad }}$ calculations}

Recently, an improved parametric method for calculating $g_{\text {rad }}$ at large optical depths was developed (Papers I and II). The improvements, as compared to the method of Alecian \& Artru (1990a,b), were made possible because of the use of OP opacities and data (Seaton et al. 1992). Once the parameters of the various ions are calculated, the parametric method has the advantage of giving relatively accurate $g_{\text {rad }}$ without having to deal with the enormous amount of atomic data normally necessary to obtain $g_{\text {rad }}$. The parameters for 12 trace elements $(\mathrm{C}, \mathrm{N}, \mathrm{O}, \mathrm{Ne}$, $\mathrm{Na}, \mathrm{Mg}, \mathrm{Al}, \mathrm{Si}, \mathrm{S}, \mathrm{Ar}, \mathrm{Ca}$ and $\mathrm{Fe}$ ) were calculated in Paper II.

The method used here to calculate the $g_{\mathrm{rad}}$ of Sc is based on this parametric form of $g_{\mathrm{rad}}$ formulae (SVP method) of both bound-bound (Alecian 1985; Alecian \& Artru 1990a; Papers I and II) and bound-free (Alecian 1994; Papers I and II) transitions. In this method, radiative accelerations due to atomic lines depend on 4 parameters for each ion (Eq. (1) of Paper II), calculated at layers where the relative population of the ion $i$ is near its maximum. The first parameter $\phi_{i}^{*}$ (Eqs. (10) to (13) of Paper I) is related to the strength of the bound-bound transitions through a weighted average of the $g f$ values. The second parameter $\psi_{i}^{*}$ (see Eq. (14) of Paper I) is related to the average width of the line profiles and controls saturation. A third parameter $\xi_{i}^{*}$ is related to the ion contribution to the total opacity (we will neglect it for Sc since it is of little importance for less abundant elements).
Meanwhile, the value of the parameter $\alpha_{i}{ }^{1}$ is determined by fitting our parametric accelerations to those obtained by a more accurate method (Seaton 1997, see Paper I for more details).

A similar parametric equation was also used for bound-free transitions (Eq. (9) of Paper II). This approximate formula is less accurate than for bound-bound transitions and needs the knowledge of energy levels for each ion and the computation of the partition functions at each model layer. Two other parameters $a_{i}$ and $b_{i}$ (correction parameters for bound-free acceleration) are determined for each ion by a same kind of fitting procedure as employed for lines. These six parameters, along with the formulae of Paper II, define the SVP method. They are calculated here, in an Am stellar model, for the 12 trace elements mentioned above. These elements are treated by Seaton (1997), and our fitting procedure is achieved using version OPCD 2.1 of the tables available at $\mathrm{CDS}^{2}$.

The accurate evaluation of radiative accelerations necessitates detailed and complete atomic data (including all strong transitions) for each element. This is still lacking for scandium. A rather large data base for energy levels can be found in the NIST Atomic Spectra Database ${ }^{3}$, but this database is incomplete for bound-bound transitions, especially in regards to transition probabilities.

As shown in Alecian \& Artru (1990b), parameters $\phi_{i}^{*}$ and $\psi_{i}^{*}$ generally vary quite monotonously along isoelectronic sequences. It is then possible to approximate these parameters for some of the ions of the elements for which atomic data are lacking, by interpolating between the nearest isoelectronic neighbours with known parameters, to the ions in question. The interpolation is done via the atomic charge variable. We have then considered that to apply this interpolation method to Sc ions, using well calculated parameters from isoelectronic neighbours, is preferable than to calculate directly the parameters, using incomplete atomic data. Also, it would be impossible to do the fitting procedure of Paper II, since the $g_{\mathrm{rad}}$ of Sc are not included in Seaton (1997) or elsewhere.

We have interpolated the parameters for the bound-bound transitions of the ions ScIII to ScXX, but not for ScII since parameters for an isoelectronic neighbour are missing (namely the parameters for CaI). The parameters which were directly evaluated by Alecian \& Artru (1990b) for ScII from atomic data were then used. Parameters $a_{i}$ and $b_{i}$ were respectively set to 1 and 0 (this corresponds to no correction). Relative populations of Sc ions and partition functions have been calculated using the atomic energy level data of the NIST Atomic Spectra Database.

\subsection{Numerical results}

The results shown in this section have been obtained for a $1.9 M_{\odot}$ stellar model with $T_{\text {eff }}=7610 \mathrm{~K}$ and an age of $807 \mathrm{Myr}$ (model named 1.9P1 in Talon et al. 2006).

To verify the accuracy of this interpolation method along isoelectronics sequences, we have simulated it for elements for which the parameters are obtained by detailed computations, and for which the SVP method is known to work well, i.e. Si and $\mathrm{Ca}$ (see Paper II). Note that for these elements, accurate radiative accelerations can also be calculated by the Seaton (1997) method, which makes the verification process especially confident. We have compared for these elements, accelerations

\footnotetext{
1 This parameter characterizes an average Voigt profile of the lines for the ion under consideration, see Eq. (1) of Paper II.

2 http://vizier.u-strasbg.fr/topbase/op.html

3 http://physics.nist.gov/PhysRefData/ASD/index.html
} 


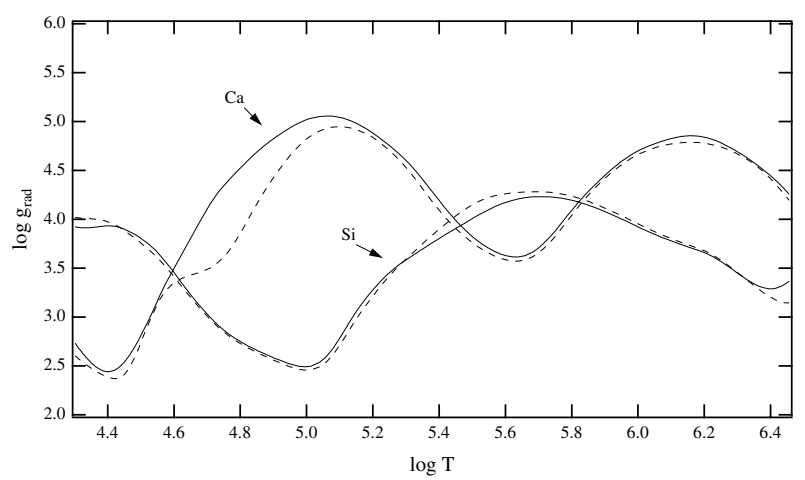

Fig. 1. Radiative accelerations of $\mathrm{Si}$ and $\mathrm{Ca}$ in a model with $T_{\text {eff }}=$ $7610 \mathrm{~K}\left(1.9 M_{\odot}\right)$. The solid lines represent the results of the SVP method using known parameters, while the dashed lines represent the $g_{\mathrm{rad}}$ using the parameters obtained by our interpolation method.

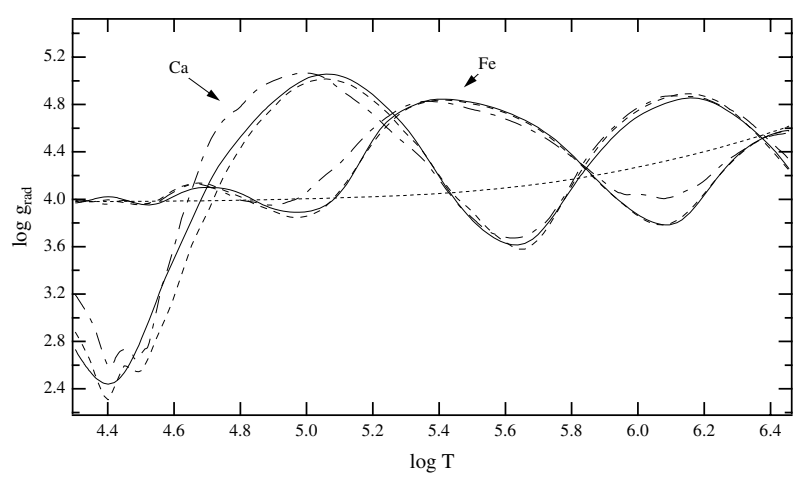

Fig. 2. Radiative accelerations of $\mathrm{Ca}$ and $\mathrm{Fe}$ using solar abundances calculated with the SVP method (solid lines) and those of Seaton (1997) (dashed lines) in the same stellar model as in Fig. 1. The dashed-dotted lines represent the accelerations from the models of Talon et al. (2006) using the local abundances obtained by the diffusion process. The local gravity is represented by the dotted curve.

obtained using their true parameters with those (assuming that they are unknown) obtained with parameters calculated by interpolation along isoelectronics sequences. Results confirmed the validity of our interpolation method, at least for $\mathrm{Si}$ and $\mathrm{Ca}$ (see Fig. 1). The parameters of some weakly ionized ions cannot be obtained by interpolation since they do not have isoeletronic neighbours with known parameters. In order to include a maximum number of ions, we have used the parameters $\phi_{i}^{*}$ and $\psi_{i}^{*}$ of SiII, CaII and CaIII from Alecian \& Artru (1990b). The results for $\mathrm{Ca}$ are not very accurate at low temperatures. This is due to the fact that the parameters $\phi_{i}^{*}$ do not, in this case, vary monotonously for weakly ionized ions (see the figures in Alecian \& Artru 1990b). The interpolation method underestimates contributions of $\mathrm{CaIV}$ and $\mathrm{CaV}$ to the acceleration. Nevertheless, deeper in the star, both methods give very similar results. Although we only study the case of Sc in this work, we consider that this interpolation method could be extended to other elements for which the atomic data are not completely known, provided that their ions have isoelectronic neighbours for which parameters are well determined.

Figure 2 shows the $\mathrm{Ca}$ and $\mathrm{Fe}$ radiative accelerations for a solar abundance using the SVP method and those obtained by applying the method of Seaton (1997) ${ }^{4}$. Also shown, are the

${ }^{4}$ With version OPCD 2.1 of data and codes available at Centre de Données de Strasbourg.

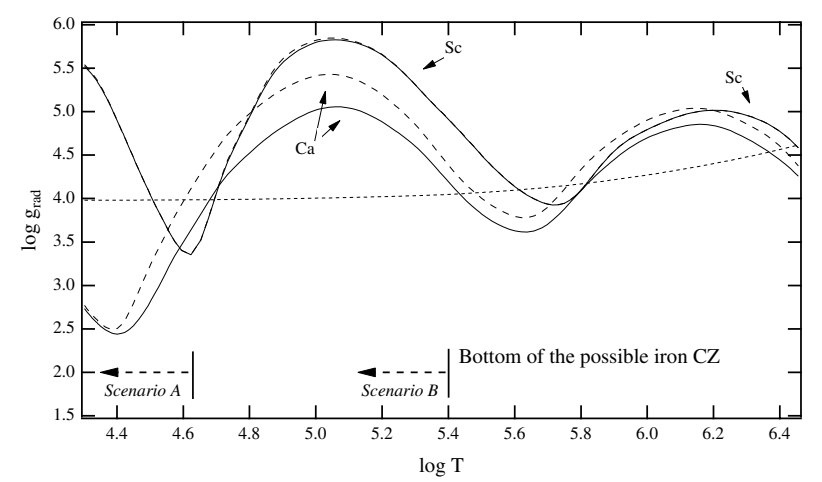

Fig. 3. Radiative accelerations of $\mathrm{Ca}$ and $\mathrm{Sc}$ for abundances of 1 (solid lines) and 0.1 (dashed lines) times their solar values in the same stellar model as in Fig. 1. The Sc accelerations are calculated by our interpolation method and those of Ca by the SVP method. Also shown are the approximate locations of the bottom of the convection zones of scenarios $A$ and $B$, and the local gravity (dotted line).

$g_{\text {rad }}$ found by Talon et al. $(2006)^{5}$. These $g_{\text {rad }}$ are calculated via the opacity sampling method (e.g. LeBlanc et al. 2000) using the local abundance obtained by the diffusion process. We may conclude, from these, that the results from the SVP method are satisfactory.

Figure 3 shows the $g_{\text {rad }}$ of Sc (calculated by the interpolation method) and $\mathrm{Ca}$ (calculated by the SVP method). The parameters for $\mathrm{Ca}$ are the same as those used for the accelerations shown in Fig. 2. The parameters for Sc are interpolated from those of $\mathrm{Ca}$ and Fe. Two abundances are shown: 1 and 0.1 times their solar values. The two valleys seen in the curves presented are related to the Ar-like and Ne-like configurations, where the $g_{\text {rad }}$ are naturally weaker. We can observe that the two curves for Sc are quite similar because this element is not very abundant and, at the abundances used here, the $g_{\text {rad }}$ are completely unsaturated and near their maximal values.

Generally, we consider that the SVP method has an accuracy of about $30 \%$ (or $0.1 \mathrm{dex}$ ) compared to detailed methods in the temperature range of interest here (Paper II). We can suppose that the accuracy is of the same order of magnitude for our $g_{\text {rad }}$ estimation for Sc. Unlike the curve shown for Ca in Fig. 1, the $g_{\text {rad }}$ of Sc at low temperatures does not seem to have abnormal behaviour. This probably means that these $g_{\mathrm{rad}}$ are relatively good throughout the model. However, only detailed $g_{\text {rad }}$ calculations could confirm this.

\section{The relative abundances of $\mathrm{Ca}$ and $\mathrm{Sc}$ in Am stars}

The principal characteristic of Am stars is the presence of $\mathrm{Ca}$ and Sc deficiencies at their surface. Several observational studies have evaluated the abundances for these two elements as well as others in Am stars (e.g. Adelman 1994; Hui-Bon-Hoa et al. 1997; Hui-Bon-Hoa \& Alecian 1998; Hui-Bon-Hoa 1999; Griffin 2002). These studies show that generally, Sc is more underabundant (relative to solar values) than $\mathrm{Ca}$ in Am stars.

When neglecting all other factors than gravity and radiative acceleration, if $g_{\mathrm{rad}}>g$ the element will be driven upwards. While if $g_{\text {rad }}<g$ the element will migrate towards the centre of the star. At first approximation, since $g_{\text {rad }}>g$ means also that

\footnotetext{
5 These accelerations were kindly communicated by O. Richard.
} 
a larger abundance of the element can be supported by the radiation field, one considers that the element should become overabundant (with respect to solar value) in those layers, and underabundant in layers where $g_{\mathrm{rad}}<g$. Of course, time-dependent diffusion, which is a non-linear process, is more complicated than what is described by this simplified approach.

As mentioned previously, two possible scenarios are suggested to explain these underabundances which both depend on the diffusion process.

Scenario A supposes that the surface abundances emanate from the regions below the superficial hydrogen convection zone, assuming a global mass flow (defined by the mass loss rate). These regions are near where the Ar-like configurations of Sc and $\mathrm{Ca}$ dominate, thus explaining their underabundance. Alecian $(1986,1996)$ showed that models with diffusion while including mass loss, can mimic a deepening of the surface mixing zone, and results in varying superficial abundances as a function of time. This study showed that the surface abundance of $\mathrm{Ca}$ and $\mathrm{Sc}$ for Am stars depend on both the mass loss rate and mixing length of convection. Time variation of superficial abundances due to mass loss was first considered qualitatively by Michaud \& Charland (1986). They used a kinematic method and have shown that mass loss can produce variations of superficial abundances. But they have not solved the time-dependent continuity equation as in Alecian (1996). Both of these models do not take into account the structural changes due to diffusion as those of Richard et al. (2001) for instance.

Scenario $B$ supposes that the surface abundances come from much deeper in the star, near where Sc and $\mathrm{Ca}$ are in the Ne-like configuration (Richard et al. 2001; Richer et al. 2000). A convection zone created by an accumulation of iron in the region where $\log T \simeq 5.3$ in these evolutionary models including atomic diffusion (but while neglecting mass loss) is at the base of this conclusion. The $g_{\mathrm{rad}}$ of Fe shown in Fig. 2 are consistent with an accumulation of this element in this region of the star. However, as previously mentioned, mass loss could modify the stratification profiles of $\mathrm{Fe}$ and of the other elements.

More recently (Michaud et al. 2005), these models were applied to the star $o$ Leo A, and lead to similar abundances as observed by Griffin (2002) for this star. These authors mentioned that this star serves as a severe test for the deep mixing framework (scenario B). They also mention that a model supposing surface abundances created below the superficial hydrogen convection zone (Watson 1971) would be diluted during the subgiant phase. This point will be discussed in the conclusion. They also state that other species that could be compared to observations would be useful.

In Fig. 3, we notice that in the region of the Ne-like configurations for $\mathrm{Ca}$ and $\mathrm{Sc}$ and where the abundance anomalies occur in scenario $B\left(\right.$ at $\log T \simeq 5.3$ ), the $g_{\text {rad }}$ of Sc are larger than those of $\mathrm{Ca}$, and even larger than gravity up to $\log T \simeq 5$.6. It is instructive to look at the curves with an abundance of 0.1 times solar values in Fig. 3, since in Am stars, both $\mathrm{Ca}$ and Sc are generally underabundant. These curves show that if scenario $B$ is the correct one, the mixing has to be deeper than $\log T=5.7$ for $\mathrm{Sc}$ to be more underabundant than $\mathrm{Ca}$ (relative to solar values). This is deeper than the mixing predicted in the models of Richard et al. (2001).

In the stellar model presented here, the bottom of the hydrogen convection zone is located at $\log T=4.3$. This is not deep enough to explain the relative underabundances of $\mathrm{Ca}$ and Sc simultaneously, if one strictly considers the original model of Watson (1971). But, this is not incompatible with Scenario A. The region where the $g_{\mathrm{rad}}$ of Sc is weaker than those of $\mathrm{Ca}$ and that is consistent with their observed relative abundances is located near $\log T=4.6$ for abundances of 0.1 times solar values. In this region, $g_{\mathrm{rad}}$ of both $\mathrm{Ca}$ and $\mathrm{Sc}$ are weaker than gravity for solar abundances. According to numerical modelling of a star not very far from the present situation, Alecian (1996) showed that, assuming mass loss and considering that the bottom of the mixing zone is uncertain, Scenario A could account for such simultaneous underabundances. However, only detailed evolutionary calculations while including diffusion will be able to determine which of the two scenarios is correct.

\section{Conclusion}

The radiative accelerations of Sc presented here shed new light on the source of Am stars' abundance anomalies and represents a new diagnostic tool to test various theoretical models. Scandium could to be a key element to better understanding these stars.

Our results rekindle the question as to where the abundance anomalies emanate from in Am stars: either from a superficial or a much deeper mixing zone. The results shown here regarding $g_{\text {rad }}$ of Sc suggest that for scenario $B$ to be able to reproduce both the observed $\mathrm{Ca}$ and $\mathrm{Sc}$ abundances, the mixing must be deeper than that found in the models of Talon et al. (2006) or those of Richard et al. (2001) for instance. In these models, the mixing goes down to approximately $\log T=5.3$, while from the results shown here, it has to go down to approximately $\log T=5.7$ to be able to reproduce both $\mathrm{Ca}$ and $\mathrm{Sc}$ observed abundances.

Relating to the question of dilution of the abundances during the subgiant phase of evolution, it is not clear if this argument can eliminate scenario A. Even though the surface underabundances are created at much shallower regions of the star in this scenario compared to scenario $B$, there should also exist an underabundance of both $\mathrm{Ca}$ and $\mathrm{Sc}$ in the two regions where the noble gas configurations dominate. The value of the overabundance that should prevail between these two regions as well as the depth of the mixing zone will determine to what extent the surface abundances are affected at the subgiant stage. Only detailed evolutionary calculations while including the deeper mixing at the subgiant stage could show how the surface abundances of scenario $A$ are affected at that stage of evolution. The predicted abundances of other elements should also give insight into the amplitude of the dilution.

Our results show that near $\log T=4.6$ (for abundances of 0.1 times solar values), the $g_{\mathrm{rad}}$ of $\mathrm{Ca}$ are stronger than those of Sc. Scenario A could then produce $\mathrm{Ca}$ and Sc abundances consistent with their relative observed abundances in Am stars. The results presented here for $\mathrm{Sc}$ thus reinforce the superficial mixing zone scenario but only evolutionary stellar models with mass loss and diffusion, while including Sc, might help to finally solve the Am stars' mysteries. We hope that the $g_{\text {rad }}$ of Sc shown here, or others calculated directly from reliable atomic data, will be included in evolutionary models in the near future. For that purpose, the parameters for Sc calculated here are made available at http: //www . umoncton.ca/leblanfn/grad.

Acknowledgements. Authors thank O. Richard for kindly communicating the stellar model used in this paper. This research was partially funded by NSERC and La Faculté des Études Supérieures et de la Recherche de l'Université de Moncton. We also thank RQCHP for computing time. One of us (F.L.) is grateful for a one month visiting position at l'Observatoire de Meudon and at l'Université de Paris 7. We acknowledge the financial support of Programme National de Physique Stellaire (PNPS) of CNRS/INSU, France. 


\section{References}

Abt, H. A. 1961, ApJS, 6, 37

Adelman, S. J. 1994, MNRAS, 271, 355

Alecian, G. 1985, A\&A, 145, 275

Alecian, G. 1986, A\&A, 168, 204

Alecian, G. 1994, A\&A, 289, 885

Alecian, G. 1996, A\&A, 310, 872

Alecian, G., \& Artru, M.-C. 1990a, A\&A, 234, 323

Alecian, G., \& Artru, M.-C. 1990b, A\&AS, 83, 379

Alecian, G., \& LeBlanc, F. 2000, MNRAS, 319, 677

Alecian, G., \& LeBlanc, F. 2002, MNRAS, 332, 891 (Paper I)

Griffin, R. E. 2002, AJ, 123, 988

Hui-Bon-Hoa, A. 1999, A\&A, 343, 261

Hui-Bon-Hoa, A., \& Alecian, G. 1998, A\&A, 332, 224

Hui-Bon-Hoa, A., Burkhart, \& Alecian, G. 1997, A\&A, 323, 901

Kurucz, R. L. 1990, Trans. IAU, Vol. XXB, ed. D. McNally (Kluwer), 168
Kurucz, R. L., \& Peytremann, E. 1975, Smithsonian Astrophys. Obs. Spce. Rep., 362

LeBlanc, F., Michaud, G., \& Richer, J. 2000, ApJ, 538, 876

LeBlanc, F., \& Alecian, G. 2004, MNRAS, 352, 1329 (Paper II)

Michaud, G. 1970, ApJ, 160, 641

Michaud, G., \& Charland, Y. 1986, ApJ, 311, 326

Michaud, G., Tarasick, D., Charland, Y., \& Pelletier, C. 1983, ApJ, 269, 239

Michaud, G., Richer, J., \& Richard, O. 2005, ApJ, 623, 442

Preston, G. W. 1974, ARA\&A, 12, 257

Rogers, F. J., \& Iglesias, C. A. 1992, ApJ, 401, 361

Richard, O., Michaud, G., \& Richer, J. 2001, ApJ, 558, 377

Richer, J., Michaud, G., \& Turcotte, S. 2000, ApJ, 529, 338

Seaton, M. J. 1997, MNRAS, 289, 700

Seaton, M. J., Zeippen, C. J., Tully, J. A., et al. 1992, Rev. Mex. Astron. Astrofis., 23,19

Smith, M. A. 1971, A\&A, 11, 325

Talon, S., Richard, O., \& Michaud, G. 2006, ApJ, 645, 634

Watson, W. D. 1971, A\&A, 13, 263 